\title{
Effects of ZnO Nanowire Length on Surface-Assisted Laser Desorption/Ionization of Small Molecules
}

\author{
Won Jik Shin, ${ }^{\mathrm{a}, \mathrm{b}}$ Jeong Ho Shin, ${ }^{\mathrm{c}, \mathrm{d}}$ Jae Yong Song, ${ }^{\mathrm{c}, \mathrm{e}}$ and Sang Yun Han ${ }^{\mathrm{a}}$ \\ ${ }^{a}$ Center for Nano-Bio Convergence Research, Korea Research Institute of Standards and Science, Daejeon, \\ Republic of Korea \\ ${ }^{\mathrm{b}}$ Department of Chemistry, Chungbuk National University, Cheongju, Republic of Korea \\ c Division of Industrial Metrology, Korea Research Institute of Standards and Science, Daejeon, Republic of \\ Korea \\ ${ }^{\mathrm{d}}$ Department of Nanomaterials Science and Technology, University of Science and Technology, Daejeon, \\ Republic of Korea \\ e Department of Nanoscience, University of Science and Technology, Daejeon, Republic of Korea
}

The effects of nanowire (NW) length on surface-assisted laser desorption/ionization (SALDI) mass spectrometry (MS) of small molecules were investigated using ZnO NWs of $50 \mathrm{~nm}$ diameter with a broad range of lengths ranging from 25 to $1600 \mathrm{~nm}$. Characterization of the $\mathrm{ZnO}$ NWs revealed that the length was the only parameter that varied in this study, while other properties of the NWs remained essentially the same as the bulk properties. Experiments on SALDI efficiency exhibited that the SALDI processes on NWs have a certain length window. In the present case of $\mathrm{ZnO}$ NWs, the SALDI efficiency was found to be enhanced on the nanowires of $250 \mathrm{~nm}$ length, corresponding to an aspect ratio of 5. The roles of NW length in the SALDI processes were discussed from the viewpoint of efficient energy-transfer media as well as physical obstacles screening laser irradiation and preventing the escape of nascent ions from NW surfaces. The existence of the length window may provide valuable insight for tailoring new nanostructures for efficient SALDI of small molecules. (J Am Soc Mass Spectrom 2010, 21, 989-992) (c) 2010 American Society for Mass Spectrometry

$\mathrm{D}$ ue to interference of matrix ion peaks in the small mass range $(<700 \mathrm{Da})$, application of the matrix-assisted laser desorption/ionization (MALDI) method has been limited for mass spectrometry of small and biologically important molecules such as drugs, metabolites, lipids, and the like. However, recent advances in nanotechnology, particularly in the development of nanomaterial fabrications, have shed light on laser desorption/ionization of small molecules without treatment of conventional matrices [1].

A variety of nanostructured materials have been examined for their capabilities of surface-assisted laser desorption/ionization (SALDI). Nano- and micrometersized particles including $\mathrm{Au}, \mathrm{Al}, \mathrm{Mn}, \mathrm{Sn}, \mathrm{W}, \mathrm{Si}, \mathrm{Sn}$, $\mathrm{SnO}_{2}, \mathrm{TiO}_{2}$, and $\mathrm{ZnO}$ [2-5] have been studied as potential SALDI matrices. NWs of $\mathrm{ZnO}, \mathrm{SnO}_{2}, \mathrm{GaN}, \mathrm{SiC}$ [6], and carbon nanotubes [7], have also been examined. Among many examined materials, Si has been a subject of extensive studies due to the discovery of the desorp-

Address reprint requests to Dr. S. Y. Han, Center for Nano-Bio Convergence Research, Korea Research Institute of Standards and Science, Daejeon 305-340, Republic of Korea and Dr. J. Y. Song, Division of Industrial Metrology, Korea Research Institute of Standards and Science, Daejeon 305-340, Republic of Korea. E-mail: sanghan@kriss.re.kr and jysong@ kriss.re.kr tion/ionization on porous silicon (DIOS) process by Siuzdak and colleagues $[8,9]$. Investigations of DIOS were further extended to Si NPs [10], Si NWs [11], and nanostructured Si surfaces $[12,13]$. Some mechanistic aspects involved in DIOS were also reported [14-17], but the DIOS mechanism is not yet fully understood.

$\mathrm{ZnO}$ is an important semiconducting material for many industrial applications [18]. It possesses a direct wide band gap $\left(\mathrm{E}_{\mathrm{g}} \sim 3.3 \mathrm{eV}\right)$, allowing absorption of $\mathrm{UV}$ photon energy at $355 \mathrm{~nm}(3.49 \mathrm{eV})$ with a large absorption coefficient $(a)$ of $1.7 \times 10^{5} / \mathrm{cm}^{-1}$. The penetration depth $(1 / a)$ of $355 \mathrm{~nm}$ photons is only $60 \mathrm{~nm}$. The bulk thermal conductivity $(\kappa)$ of $\mathrm{ZnO}$ has been reported to range from $0.6 \sim 1.4 \mathrm{~W} / \mathrm{cm} \mathrm{K}$, which is quite low and comparable to that of $\mathrm{Si}$, i.e., $1.5 \mathrm{~W} / \mathrm{cm} \mathrm{K}$. Preparation of $\mathrm{ZnO}$ as various nanostructures including nanotubes, nanorings, and nanobelts has also been well documented.

Previously, ZnO NPs and NWs have been successfully demonstrated as SALDI matrices. Using ZnO NPs with an average diameter of $350 \mathrm{~nm}$, Watanabe et al. showed successful laser desorption/ionization of various small molecules such as drugs, oligosaccharides, lipids, and synthetic polymers [5]. Kang et al. demonstrated the SALDI of small peptides on $\mathrm{ZnO}$ NWs with diameters of $50-100 \mathrm{~nm}$ and lengths of tens of microme- 
ters [6]. ZnO NWs showed better SALDI performance than the other NWs examined in their work. In this work, we investigated the effects of nanowire size, particularly in terms of length, on the SALDI efficiency for small drug molecules using varied lengths of constant diameter $\mathrm{ZnO} \mathrm{NW}$, ranging from 25 to $1600 \mathrm{~nm}$, while keeping other properties as the same as the bulk, single crystal properties.

\section{Experimental}

\section{Fabrication of $\mathrm{ZnO} \mathrm{NWs}$}

$\mathrm{ZnO} \mathrm{NWs}$ were produced on an $\mathrm{Au} / \mathrm{Ti} / \mathrm{Si}$ structured substrate by a vapor transport process. The detailed experimental procedure can be found elsewhere [19]. The lengths of $\mathrm{ZnO} N W$ s were determined in a range of 25 to $1600 \mathrm{~nm}$ by controlling the growth time.

\section{SALDI Experiments}

The test drug molecules, clonidine (exact mass: 230.1 Da), tamoxifen (371.5 Da), ketoconazole (531.4 Da), and azithromycin (749.0 Da) were purchased from SigmaAldrich (Yongin, Korea) and used without further purification. Single crystal $\mathrm{ZnO}$ substrates with (0001) direction were purchased from MTI Corporation. (Richmond, CA, USA).

In a typical SALDI experiment, a $0.5 \mu \mathrm{L}$ drop of 10 $\mu \mathrm{M}$ solution in $10 \% \mathrm{MeOH}$ in ultrapure water (5 pmol) was pipetted onto a $\mathrm{ZnO} \mathrm{NW}$ substrate. After drying under ambient conditions, SALDI experiments were performed using a MALDI mass spectrometer (Autoflex III; Bruker-Daltonics, Bremen, Germany) equipped with a high-repetition UV laser $(355 \mathrm{~nm})$. We report the laser power in terms of percentage laser power, estimated from the attenuator setting. The ion yields were obtained at least quadruply, and the average and standard deviations were calculated from the measured peak intensities.

\section{Results and Discussion}

\section{Characterization of $\mathrm{ZnO}$ NWs with Various Lengths}

The fabricated $\mathrm{ZnO}$ NWs were routinely examined using SEM and XRD (see Supplementary Data, which can be found in the electronic version of this article). The SEM images revealed that the $\mathrm{ZnO}$ NWs had a diameter of $50 \mathrm{~nm} \pm 10 \%$, irrespective of their lengths. The number density of NWs was estimated from a top view SEM image to be about $1.5 \times 10^{10} \mathrm{~cm}^{-2}$, which gives $83 \mathrm{~nm}$ for the average center-to-center distance and thus $33 \mathrm{~nm}$ for the average closest spacing between adjacent NWs. As the lengths of the NWs increase, the surface area is accordingly expanded.

The observed XRD patterns clearly indicate that the ZnO NWs of this study carry a good single crystallinity, having a WZ (würztite) crystal structure characteristic of bulk $\mathrm{ZnO}$ crystal. Further photoluminescence experiments preformed for the 50 and $250 \mathrm{~nm}$ long $\mathrm{ZnO}$ NWs gave essentially the same results, revealing two optical transitions that are known for the crystalline $\mathrm{ZnO}$ spectrum: the A free excitons and their first excitedstate transitions at 3.38 and $3.42 \mathrm{eV}$, respectively. In the case of Si NWs, it was in fact previously reported that the quantum confinement effects would become significant when the diameter of $\mathrm{Si}$ NWs becomes smaller than $2.2 \mathrm{~nm}$ [20]. Therefore, it can be assumed that the change of length in this study may not alter the material properties from those of bulk crystals.

\section{Near-Threshold SALDI Behaviors}

Figure 1 displays the SALDI mass spectra taken for the four molecules on the $250 \mathrm{~nm}$ long $\mathrm{ZnO}$ NWs. The SALDI on ZnO NWs produced strong intact ions. In the ionization, the charge was acquired by protonation or cationization by $\mathrm{Na}^{+}$and $\mathrm{K}^{+}$, which might be residuals from the former synthesis steps of NWs, the drug solution, or the environment. Other peaks in the mass spectra most likely originated from sample contamination rather than fragmentation. On the $250 \mathrm{~nm}$ long $\mathrm{ZnO}$ NWs, 5 pmol loading to a $1 \mathrm{~mm}$ diameter circle corresponds to a surface density of $4.7 \times 10^{13} \mathrm{~cm}^{-2}$ for drug molecules. This gives a number density that is lower than the typical surface atom density of $\sim 10^{15}$. It was thus assumed that the surface coverage of the sample would not exceed a monolayer in this work.

We first investigated the threshold ionization behaviors using $\mathrm{ZnO}$ NWs with different lengths by measuring ion intensities as a function of laser power (see Supplementary Data). However, due to low ion intensities in the threshold region, it was difficult to assess the true onsets of SALDI signals. Instead, we chose to evaluate the laser power needed to obtain an ion signal intensity of 3000 in arbitrary unit (a.u.), which corresponded to a signal-to-noise ratio of $\sim 100$, and was slightly above the true threshold. The near-threshold laser powers defined in this way are presented in Figure 2a. From the results, it is evident that the SALDI process has an apparent length dependence and appears to be most efficient on the $\mathrm{ZnO}$ NWs with a length of $250 \mathrm{~nm}$. For comparison, we also carried out the same experiment on a single crystal $\mathrm{ZnO}$ substrate. The nearthreshold laser power for the $\mathrm{ZnO}$ crystal was measured to be $61 \%$, which is about one and a half of that for the $250 \mathrm{~nm}$ long $\mathrm{ZnO}$ NWs.

We further confirmed the efficient SALDI process on the $250 \mathrm{~nm}$ long $\mathrm{ZnO}$ NWs, by measuring ion yields at an increased and fixed laser power of $48 \%$ for the $\mathrm{ZnO}$ NWs with different lengths (Figure 2b). The laser power lies substantially above the threshold, where, for example, the SALDI ion intensity on the $250 \mathrm{~nm}$ long $\mathrm{ZnO}$ NWs was several times greater than the near-threshold ionization. As shown in Figure $2 b$, the results obtained using four drug molecules all support that the most 


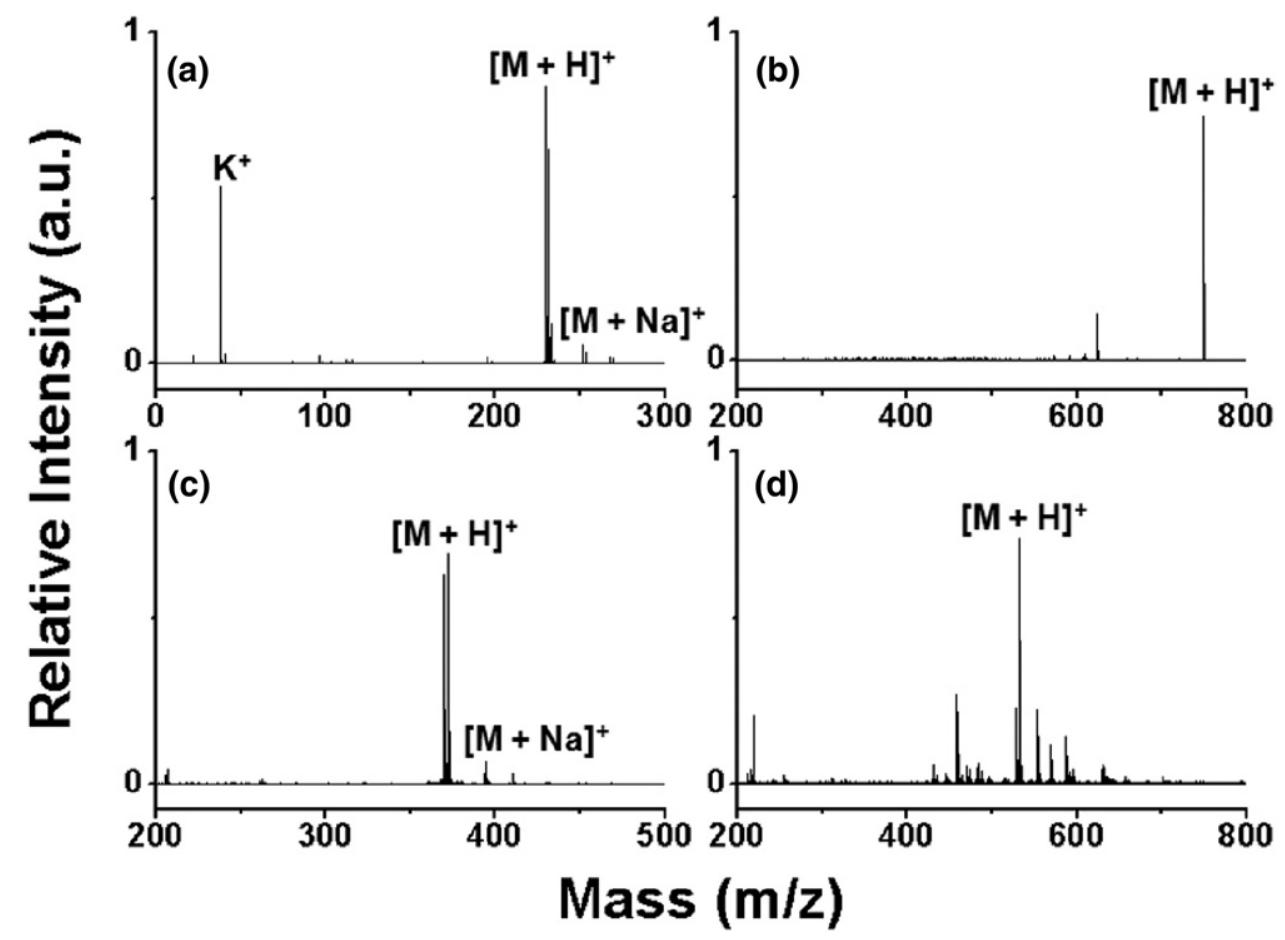

Figure 1. SALDI mass spectra obtained for 5 pmol of (a) clonidine (exact mass: $230.1 \mathrm{Da}$ ), (b) azithromycin (749.0), (c) Tamoxifen (371.5), (d) Ketoconazole (531.4) using the ZnO NWs of $250 \mathrm{~nm}$ length.

pronounced SALDI processes occurred on the $250 \mathrm{~nm}$ long ZnO NWs. The SALDI efficiency is then greatly lowered with longer NWs. The sensitivity of SALDI on the $220 \mathrm{~nm}$ long $\mathrm{ZnO}$ NWs was estimated to be better than $100 \mathrm{fmol}$ (see Supplementary Data).

\section{The Effects of NW Length on SALDI}

The results demonstrate that the SALDI process on $\mathrm{ZnO}$ NWs of $50 \mathrm{~nm}$ diameter has a certain length window where it becomes most efficient. To elucidate the actual roles of NW length in SALDI, we considered the mechanistic aspects. The noticeable difference in the SALDI efficiencies of NWs and single crystal surface indicates that the nanostructure is essential for the SALDI process. The lower threshold laser power for NWs suggests that the nanostructure may contribute to absorbing and transferring UV laser energy to molecules much more efficiently than flat surfaces. In this regard, one can consider that surface heating, which is greatly enhanced with the formation of NW structures, is a key component of SALDI processes.

In the case of $\mathrm{ZnO}$, the depth of conductive heating $(D)$ during laser irradiation $(t)$ of $7 \mathrm{~ns}$, given by $D=$ $\left(\kappa t / C_{p} \pi\right)^{1 / 2}$, is about $130 \mathrm{~nm}$, where $\kappa$ and $C_{p}$ denote thermal conductivity and heat capacity, respectively [14]. The estimated depth is greater than the NW diameter of $50 \mathrm{~nm}$, and thus the heat conduction in the transverse direction is limited by the surface boundary condition. In addition, the absorption of $355 \mathrm{~nm}$ may take place efficiently through the entire NW, as the UV penetration depth $(1 / a=60 \mathrm{~nm})$ is comparable to the NW diameter $(50 \mathrm{~nm})$, contributing to efficient utilization of laser energy in the $\mathrm{ZnO}$ NWs. The heat accumulated in the NWs can then drastically increase the surface temperature and promote desorption of molecules in the SALDI processes. For example, surface heating of Si by UV laser absorption can increase its surface temperature up to $1000 \mathrm{~K}$ [14]. Under these circumstances, if the length of the NWs is much shorter than the depth of conductive heating $(D)$, the absorbed energy may flow out through the substrate. Considering these effects, the NW lengths should be longer than the order of the depth of conductive heating $(D)$, i.e., $130 \mathrm{~nm}$ in the $\mathrm{ZnO}$ case, to enhance SALDI processes.

It is also necessary to consider the geometrical parameters involved in the $\mathrm{ZnO} \mathrm{NW}$ arrays. A NW can efficiently block the UV radiation, casting a shadow on the next NW. In addition, due to the instrumental geometry, the UV laser enters at a certain angle from the surface normal $\left(\sim 30^{\circ}\right)$. Considering that the average closest spacing between NWs is about $33 \mathrm{~nm}$, a NW can be irradiated only for about $60 \mathrm{~nm}$ from the top when it is assumed to be fully screened by a neighboring NW. We also examined the sample distribution over the $\mathrm{ZnO}$ NWs by energy dispersive spectroscopy (EDS) experiments. The EDS mapping results revealed that the sample was evenly deposited along the longitudinal direction of the NWs (see Supplementary Data). As the sample to be mass-analyzed is located between densely standing NWs with increasing NW length, it is more difficult for nascent ions to escape into the vacuum. 

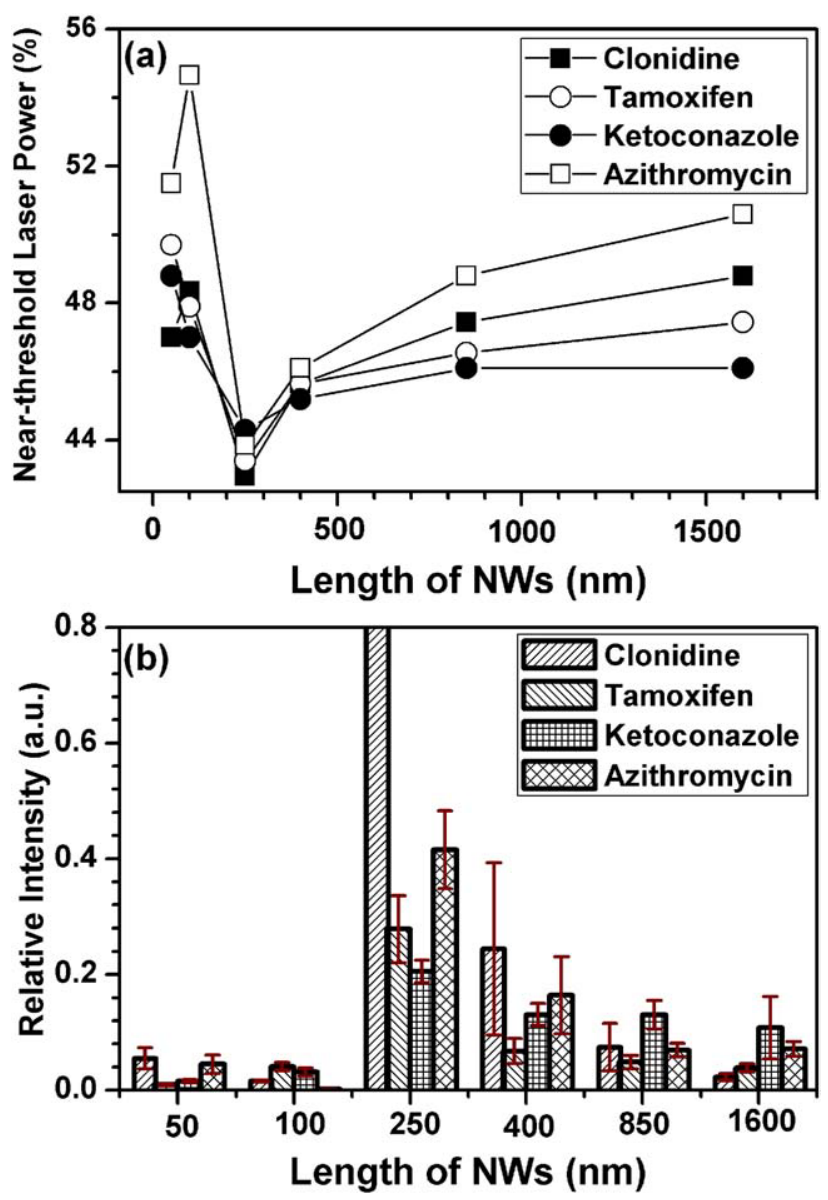

Figure 2. (a) Plots of near-threshold laser powers as a function of NW lengths obtained for four drug molecules. (b) Plots of ion intensities as a function of NW lengths obtained at a fixed laser power of $48 \%$.

Likewise, long NWs can act as a physical barrier against both laser irradiation and the escape of molecules.

\section{Conclusions}

The results of this work suggest the nanostructure is indeed essential for efficient SALDI process, in which surface heating may be deeply involved. The existence of the length window may provide valuable insights for tailoring new nanostructures for efficient SALDI of small molecules.

\section{Acknowledgments}

The authors acknowledge support for this research by the Converging Research Center Program through the NRF funded by MEST (2009-0082230). This work was also supported by KRCF through the project of Development of Characterization Techniques for Nanomaterials Safety.

\section{Appendix A Supplementary Material}

Supplementary material associated with this article may be found in the online version at doi:10.1016/ j.jasms.2010.01.030.

\section{References}

1. Guo, Z.; Ganawi, A. A. A.; Liu, Q.; He, L. Nanomaterials in Mass Spectrometry Ionization and Prospects for Biological Applications. Anal. Bioanal. Chem. 2006, 384, 584-592.

2. McLean, J. A.; Stumpo, K. A.; Russell, D. H. Size-Selected $(2-10 \mathrm{~nm})$ Gold Nanoparticles for Matrix Assisted Laser Desorption Ionization of Peptides. J. Am. Chem. Soc. 2005, 127, 5304-5305.

3. Kinumi, T.; Saisu, T.; Takayama, M.; Niwa, H. Matrix-Assisted Laser Desorption/Ionization Time-of-Flight Mass Spectrometry Using An Inorganic Particle Matrix for Small Molecule Analysis. J. Mass Spectrom. 2000, 35, 417-422.

4. Lee, K.-H.; Chiang, C.-K.; Lin, Z.-H.; Chang, H.-T. Determining Enediol Compounds in Tea Using Surface-Assisted Laser Desorption/Ionization Mass Spectrometry with Titanium Dioxide Nanoparticles Matrices. Rapid Commun. Mass Spectrom. 2007, 21, 2023-2030.

5. Watanabe, T.; Kawasaki, H.; Yonezawa, T.; Arakawa, R. SurfaceAssisted Laser Desorption/Ionization Mass Spectrometry (SALDI-MS) of Low Molecular Weight Organic Compounds and Synthetic Polymers Using Zinc Oxide (ZnO) Nanoparticles. J. Mass Spectrom. 2008, 43, 1063-1071.

6. Kang, M.-J.; Pyun, J.-C.; Lee, J.-C.; Choi, Y.-J.; Park, J.-H.; Park, J.-G.; Lee, J.-G.; Choi, H.-J. Nanowire-Assisted Laser Desorption and Ionization Mass Spectrometry for Quantitative Analysis of Small Molecules. Rapid Commun. Mass Spectrom. 2005, 19, 3166-3170.

7. Xu, S.; Li, Y.; Zou, H.; Qiu, J.; Guo, Z.; Guo, B. Carbon Nanotubes as Assisted Matrix for Laser Desorption/Ionization Time-of-Flight Mass Spectrometry. Anal. Chem. 2003, 75, 6191-6195.

8. Wei, J.; Buriak, J. M.; Siuzdak, G. Desorption-Ionization Mass Spectrometry On Porous Silicon. Nature 1999, 399, 243-246.

9. Shen, Z.; Thomas, J. J.; Averbuj, C.; Broo, K. M.; Engelhard, M.; Crowell J. E.; Finn, M. G.; Siuzdak, G. Porous Silicon as a Versatile Platform for Laser Desorption/Ionization Mass Spectrometry. Anal. Chem. 2001, 73, 612-619.

10. Wen, X.; Dagan, S.; Wysocki, V. H. Small-Molecule Analysis with Silicon-Nanoparticle-Assisted Laser Desorption/Ionization Mass Spectrometry. Anal. Chem. 2007, 79, 434-444.

11. Go, E. P.; Apon, J. V.; Luo, G.; Saghatelian, A.; Daniels, R. H.; Sahi, V.; Dubrow, R.; Cravatt, B. F.; Vertes, A.; Siuzdak, G. Desorption/Ionization on Silicon Nanowires. Anal. Chem. 2005, 77, 1641-1646.

12. Finkel, N. H.; Prevo, B. G.; Velev, O. D.; He, L. Ordered Silicon Nanocavity Arrays in Surface-Assisted Desorption/Ionization Mass Spectrometry. Anal. Chem. 2005, 77, 1088-1095.

13. Northen, T. R.; Yanes, O.; Northen, M. T.; Marrinucci, D.; Uritboonthai, W.; Apon, J.; Golledge, S. L.; Nordstrom, A.; Siuzdak, G. Clathrate Nanostructures For Mass Spectrometry. Nature 2007, 449, 1033-1037.

14. Alimpiev, S.; Nikiforov, S.; Karavanskii, V.; Minton, T.; Sunner, J. On The Mechanism Of Laser-Induced Desorption-Ionization of Organic Compounds From Etched Silicon and Carbon Surfaces. J. Chem. Phys. 2001, 115, 1891-1901.

15. Kruse, R. A.; Li, X.; Bohn, P. W.; Sweedler, J. V. Experimental Factors Controlling Analyte Ion Generation in Laser Desorption/Ionization Mass Spectrometry on Porous Silicon. Anal. Chem. 2001, 73, 3639-3645.

16. Luo, G.; Chen, Y.; Daniels, H.; Dubrow, R.; Vertes, A. Internal Energy Transfer in Laser Desorption/Ionization from Silicon Nanowires. J. Phys. Chem. B 2006, 110, 13381-13386.

17. Xiao, Y.; Retterer, S. T.; Thomas, D. K.; Tao, J.-Y.; He, L. Impacts of Surface Morphology on Ion Desorption and Ionization in Desorption Ionization on Porous Silicon (DIOS) Mass Spectrometry. J. Phys. Chem. C 2009, 113, 3076-3083.

18. Ozgur, U.; Alivov, Y. I.; Liu, C.; Teke, A.; Reshchikov, M. A.; Dogan, S.; Avrutin, V.; Cho, S.-J.; Morkoc, H. A Comprehensive Review of ZnO Materials and Devices. J. Appl. Phys. 2005, 98, 041301.

19. Shin, J. H.; Song, J. Y.; Park, H. M. Growth of ZnO Nanowires on a Patterned Au Substrate. Mater. Lett. 2009, 63, 145-147.

20. Zhao, X.; Wei, C. M.; Yang, L.; Chou, M. Y. Quantum Confinement and Electronic Properties of Silicon Nanowires. Phys. Rev. Lett. 2004, 92, 236805. 